\title{
IPRM
}

PublishedOnline:01/06/2021

(https://journals.unza.zm/index.php?journal=medicine)

DOI: $10.21617 /$ jprm2021.321

ISSN: 2415-038X (Print)

\section{EDITOR'S COMMENT}

\section{The Infodemic of COVID-19 Vaccines: Science and Pseudoscience Highlights}

\author{
By: $\quad$ Brian Chanda Chiluba \\ Editor-in-Chief, JPRM \\ Lecturer and Researcher, the University of Zambia, Zambia. \\ UJMT-Fogarty Global Health Fellow \\ University of North Carolina, \\ Chapel Hill, \\ United States of America. \\ brian.chiluba@unza.zm \\ brian.chiluba@med.unc.edu
}

To cite: Chiluba BC,. The Infodemic of COVID-19 Vaccines: Science and Pseudoscience Highlights. JPRM 2021, 3(2): 1-4. doi: 10.21617/jprm2021.321

In previous editorials of this journal we first wrote "Zambia: Editorial Comment - COVID-19 Epidemiological Thought on why Politics and Religion are Compromising the Fight" [1]. We later wrote a proceeding editorial "COVID-19 Pandemic: Where Does It Stand in the Pantheon of Deadly Infectious Diseases" [2]. It is key that we keep the momentum to provide information through editorial works given that COVID19 pandemic still rages on. The observation is that there has been so much misinformation about the COVID-19 pandemic since it's outbreak. To make this even worse, much uncertainty arose due to misinformation, when vaccines began to be rolled out globally. The misinformation around COVID-19 vaccines pose an imminent threat especially in settings such as Asia and Africa. If this is not adequately addressed, the dynamic of increased infections of COVID-19 may become too much to manage in settings of especially Asia and Africa.

Since we are discussing COVID-19 vaccines, it is also important to fundamentally state from the onset that vaccine immunity is dramatically superior to natural immunity. We are stating this because, the most consistent misinformation which is axiomatic among anti-vaxxers is that natural immunity is superior to vaccine provided immunity. History offers many examples of this basic fact. Approximately one third of the Eurasian population (75-200 million) succumbed to the Black Death in the mid 1300's. No doubt the survivors had robust immunity to the particular strain of Yersinia pestis that was circulating at the time. A vaccine against plague, had it existed, might have resulted in less robust immunity among survivors, but there would have been tens of millions of more survivors. In the recent past, the Spanish flu epidemic in 1918 infected an estimated 500 million people and killed 50-100 millions of them. Perhaps the people who survived had more robust immunity to the particular strain of influenza thereafter than a vaccine, had it existed, would have provided. But if a vaccine had existed, a substantial proportion of the 50-100 million who died would have survived. Vaccine immunity led to the extinction of smallpox, a feat that natural immunity never even approached. Cases of such "mild" childhood diseases as pertussis dropped from more than 147,000 per year to less than 7,000. Annual measles cases dropped from more than 503,000 to less than 100. Diphtheria, polio and congenital rubella nearly disappeared. And if you don't get the diseases, you can't die from the diseases, can't be rendered paralyzed, blind or deaf by the diseases [3].

Insisting that the purported superiority of natural immunity among survivors means that vaccines are unnecessary makes as much sense as insisting that diet and exercise are unnecessary to prevent heart disease because most survivors won't go on to have a second 
heart attack. You have to survive the first attack for any concerns about a second heart attack to be relevant. Similarly, you have to survive an infection in order for your immune status thereafter to be relevant.

Perhaps it is also key to lay perspective on the obvious of science. It is important to know that once you get the vaccine (or a natural infection), the vaccines introduce the body to the antigens of a given pathogen (usually via a dead or weakened form of the pathogen), along with an adjuvant to stimulate your immune system. This causes the adaptive immune system to mount a response even though one is not actually under attack. Thus, the body produces the specialized cells that engage to fight a given disease, even though one does not have the disease.

The fundamental concept of a vaccine would be thought of like scouting reports from spies that inform generals about the enemy's plans and movements before the enemy attacks, thus allowing them to plan an appropriate defense. Vaccines train the adaptive immune system to be ready to fight a disease before encountering the disease. This is an important perspective of science and an important point to echo in this editorial. Most of the lay people and more often including the qualified health experts that are afraid to fact check information have been thinking a vaccine is a treatment or a cure. Ideally being vaccinated means that the body will reserve or maintain for you some specialized agents that could fight the same disease in case of a future infection, in the same way it does for other infections. This is the fundamental concept that is actually used to protect ourselves from disease. Vaccines don't protect, but the immune cells that it provokes does. This simply means that vaccines train the immune system to recognize and fight pathogens before being exposed to them. Think of vaccines as your gym trainer, training you to fight back your abusive husband. The gym trainer will only help you build the necessary skills to use in the fight, but will not fight for you in that fight.

It is key to discuss developmental processes also of vaccines. This is because most of the misinformation ask questions on the length of time it took to develop COVID-19 vaccines. Basically, there is a couple of ways vaccines are developed, there are about 3 fundamental ways of how vaccines are made, whichever way, the ultimate goal of vaccines is to stimulate your own immune system like earlier indicated. First and foremost, historically we have always cultured a virus, meaning the virus is killed and inactivated and kind of mashed up and then injected in a purified way into the body. In this way the body is exposed to particles that are not infectious and the body mount an immune response. The body will then remember that immune response, so that the next time one is exposed to a real infection, the immune system will quickly knock it out of commission. The second way vaccines are made is by making a virus less dangerous. In other words, the virus is somehow modified or attenuated so that it doesn't cause actual disease but it can still replicate and generate a pretty strong immune response. The third and last way vaccines are made is to actually purify a protein component of the virus and produce just that protein component. This is then injected in the body to generate the immune response. These 3 ways of making vaccines are the classic ways of doing it and these 3 ways have been studied for a long time. From these 3 ways for example, the chickenpox vaccine which is a live attenuated vaccine, the influenza vaccine which is the injected version were made [4].

After establishing 3 classical ways in which vaccines are made brings us to some of the first revolutionary successes of COVID-19 vaccines that have been made, the mRNA vaccines like Pfizer and Moderna. It is important to understand that mRNA COVID vaccines don't use a traditional antigen. They rather, use a fragment of mRNA for a viral protein, which causes the body to produce that protein (note: producing a single protein is very different from producing a functioning virus and does not carry any risk of infecting anyone with COVID-19; also, mRNA cannot replicate itself; it only instructs cells to produce a protein). Thus, this vaccine makes the body produce the antigen (for a short time), rather than the vaccine the other way where a vaccine is directly injected in the body with the antigen. In this way once the body produces the antigen, the immune system follows the steps described for conventional vaccines [5].

Now a lot of the Zambian population a few weeks ago received the AstraZeneca vaccine. Foremost, it's really important that people that have been vaccinated still continue to wear a mask, social distance, wash hands, etc. Let me try to explain why this should be so before pseudoscience provides falsehood. We know the vaccine protects against disease. It is also known from the efficacy of the vaccines and antibody studies thus far. In other words, when one gets vaccinated, the body will know how to fight the virus and the immune system will not get overwhelmed and go crazy or someone will not may not get fever, respiratory issues, and potentially get to be in the ICU and we have juggled around this over and over in this write up. It is also important to note that a vaccinated person can still host the disease and continue to spread it. In other words, if one gets the vaccine and comes in contact with COVID19 , such a one MIGHT be able to still harbor the virus. If such an individual came into contact with someone else who doesn't have the vaccine, they may infect them. This is a worst-case scenario. It can just be hoped that one acquires a sterilizing immunity, in which the COVID-19 antibodies protect from both disease (ie symptoms) and transmission. There is no consensus reached on this thus far, and this is because not all vaccines produce sterilizing immunity, but they don't need to in order to be effective at preventing disease (i.e. symptoms and illness). For example, the polio vaccine does not induce a sterilizing immune response but is still $90 \%$ effective. On the other hand, the HPV vaccine does prevent transmission. This is why it is very important to in the 
meantime, continue public health mitigation measures if one gets the vaccine. In short, we can't have a mask bonfire yet [6].

Now let's look at some of the existing or emerging pseudo-science surrounding COVID-19 vaccines. One of the misconceptions is that the COVID19 vaccine will induce infertility. This is a baseless claim. And, unfortunately, the claim is made in a few complicated scientific terms, making it the most dangerous form of misinformation. In a nutshell, the COVID-19 vaccine leads to the development of antibodies. If you come in contact with COVID-19 virus after getting the vaccine, these antibodies will attach to the spike on the COVID-19 virus. Once attached, the virus is no longer able to attack the body. The misinformation claims that COVID-19 antibodies also attach to spikes (called proteins) on the placenta (called syncytins).

It is key to know that infertility is a common fear tactic of anti-vaxxers. This has been seen over and over again. In fact, polio is still prevalent in some developing countries due to widespread fear that polio vaccines cause infertility. We, actually, should not be surprised that this is the next wave of pandemic misinformation. Misinformation is becoming almost as predictable as the disease itself. Secondly, those that are "naturally" infected with COVID-19 also have antibodies. If this misinformation were true, we would be seeing a higher than average early pregnancy loss in the community overall. We are not. Although COVID-19 seems to be a more severe disease in pregnancy, all complications happen later in pregnancy (particularly from lung function, not the placenta). There are a few case reports discussing early pregnancy losses in patients with COVID-19. But case reports are just one or two people. They just tell us that they co-occurred, not that they are related. There is no strong evidence that COVID-19 antibodies increase early pregnancy loss among symptomatic or asymptomatic women. The spike proteins on the placenta and on the virus are in the same family, so they work the same way. HOWEVER, they aren't similar enough (i.e. they don't have enough amino acid sequences) to cause confusion to the immune system. In other words, the immune system cannot and will not mistake the placenta for the virus. IN ADDITION, it's important to note that COVID19, itself, is affecting women's and men's reproductive health. We DO have the evidence for this. Not to mention that COVID19 also causes chronic inflammation, which is also associated with decreased fertility. So, getting COVID19 is certainly not a great second choice if you don't get the vaccine

In Zambia and Africa in general, it is unfortunate that much less attention has focused on informing, and preparing, the public on what to expect once you actually get the vaccine. This is a mistake. It is key to be transparent about what people should expect. Let's delve into this, after a vaccination, the majority if not all of the vaccinated individuals will experience a local symptom which includes pain at injection site, redness, and some swelling. I don't think anyone should be surprised about these, considering the vaccination comes with a needle. A much smaller amount of people will experience intense (defined as preventing daily activities) side effects including fatigue, muscle pain, joint pain and headache. Some will feel a severe hangover or "mild flu symptoms" and others may experience a fever. It is important as you wait for you next dose of the vaccine that the chances of side effects are higher when you get the second dose. Also note that there are no severe events that have been reported so far in Zambia and Africa of those that have taken a vaccine.

Important to note that while COVID-19 kills 1 in 200 people infected and the possibility of returning to a normal life, getting vaccinated should be a small sacrifice to pay. Among the basic and necessary premises of pseudoscience are vaccinations; are unnecessary/do more harm than good, are all about money, companies have bought thousands of scientists, doctors, health organizations and regulators to entice them to push for vaccines.

I conclude by saying that hesitation about taking a vaccine is not as sexy as a mutation, we have already had a multiple of mutations leading to the current Indian variant. If there is an imminent threat to the COVID-19 vaccine, it is the hesitation to be vaccinated. Africa and other Asians countries are yet to feel the full impact given this hesitation for a vaccine. It should also be a scientific assurance that vaccine immunity led to the extinction of smallpox, a feat that natural immunity never even approached. Everyone who is eligible should get be vaccinated as soon as they are able. Consider this as my scientific opinion.

\section{REFERENCES}

1. Chiluba, B.C. Shula, H. Zambia: Editorial Comment COVID-19 - Epidemiological Thought on why Politics and Religion are Compromising the Fight. J of Prev and Rehab Med, Vol. 2, No. 1, 2020, pp. 1- 4. doi: 10.21617/jprm2020.21.

2. Chiluba BC,. COVID-19 Pandemic: Where Does It Stand in the Pantheon of Deadly Infectious Diseases. JPRM 2021,3(1):1-3. doi: 10.21617/jprm2021.311.

3. Plague (Black Death) bacterial infection information and facts [Internet]. Science. 2021 [cited 26 May 2021]. Available-from:

https://www.nationalgeographic.com/science/article/theplague 
4. Vaccine Types [Internet]. HHS.gov. 2021 [cited 26 May 2021]. Available from:

https://www.vaccines.gov/basics/types

5. Widge A, Rouphael N, Jackson L, Anderson E, Roberts P, Makhene $\mathrm{M}$ et al. Durability of Responses after SARS-CoV-

2 mRNA-1273 Vaccination. New England Journal of

Medicine. 2021;384(1):80-82.

6. [Internet]. Fda.gov. 2021 [cited 26 May 2021]. Available

from: https://www.fda.gov/media/144453/download 